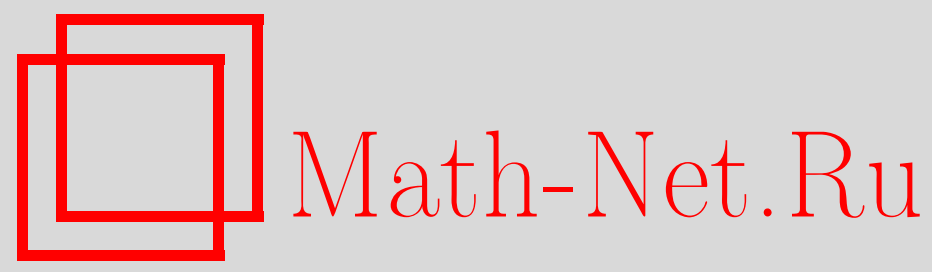

А. Ф. Измаилов, Теоремы о представлении семейств нелинейных отображений и теоремы о неявной функции, $M a-$ тем. заметки, 2000, том 67, выпуск 1, 57-68

DOI: https://doi.org/10.4213/mzm814

Использование Общероссийского математического портала Math-Net.Ru подразумевает, что вы прочитали и согласны с пользовательским соглашением http://www.mathnet.ru/rus/agreement

Параметры загрузки:

IP: 54.198 .64 .247

26 апреля 2023 г., $14: 26: 44$

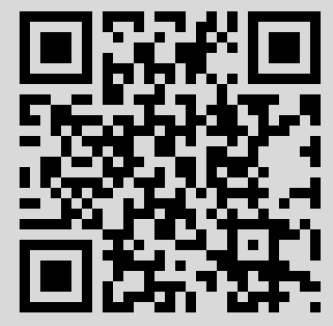




\title{
ТЕОРЕМЫ О ПРЕДСТАВЛЕНИИ СЕМЕЙСТВ НЕЛИНЕЙНЫХ ОТОБРАЖЕНИЙ И ТЕОРЕМЫ О НЕЯВНОЙ ФУНКЦИИ
}

\section{А. Ф. Измаилов}

\begin{abstract}
Приводится результат о локальном представлении семейства нелинейньх отображений в окрестности 2-регулярной особенности. Теоремы такого рода, являясь специальными формами теорем о неявной функции, содержат в себе многие важнейшие результаты локального нелинейного анализа.
\end{abstract}

Библиограффия: 7 названий.

1. Введение. Центральную роль в нелинейном анализе играют различные теоремы о неявной функции (см., например, $[1$, с. 161, 166]), в основе которых лежит идея линеаризации. Точнее, такие теоремы равносильны соответствующим результатам о приведении гладкого семейства нелинейных отображений в окрестности регулярной точки к линейному виду. Если же условия регулярности не выполнены, то результаты такого рода теряют силу: первьй дифференциал при этом не может служить сколь-нибудь адекватной аппроксимацией нелинейного отображения.

Настоящая статья является развитием работы [2], в которой, в частности, получен ряд теорем о приведении нелинейного отображения в окрестности особенности определенного класса к линейно-квадратичному виду. Здесь предлагается более общий результат о представлении параметрических семейств нелинейных отображений, который в совокупности с теоремой о существовании неявной функции из [3] содержит в себе более общую теорему о существовании неявной функции для 2-регулярных отображений.

Оговорим некоторые обозначения и термины. Пусть $B_{X}(x, \delta)=\{\xi \in X \mid$ $\|\xi-x\|<\delta\}-$ открытьй шар в линейном нормированном пространстве $X$ с центром в точке $x \in X$ радиуса $\delta>0, I_{X}$ - тождественньй оператор в $X$. Определим расстояние

$$
\rho_{X}(x, M)=\inf \{\|x-\xi\| \mid \xi \in M\}
$$

от точки $x \in X$ до множества $M \subset X$. Через $T_{x} M$ обозначим касательный конус (множество касательных векторов $[4$, с. 40]) к множеству $M$ в точке $x \in M$.

Для линейных нормированных пространств $X$ и $Y$ через $\mathcal{L}(X, Y)$ будем обозначать пространство непрерывных линейных операторов, действующих из $X$ в $Y$, а через $\mathcal{L}^{2}(X, Y)$ - пространство непрерывных билинейных отображений, действующих из

Работа выполнена при финансовой поддержке Российского фонда фундаментальных исследований, гранты № 97-01-00188 и № 99-01-00472. 
$X \times X$ в $Y$. Действие отображения $B \in \mathcal{L}^{2}(X, Y)$ на пару $\left(x^{1}, x^{2}\right) \in X \times X$ обозначается через $B\left[x^{1}, x^{2}\right]$. Каждому элементу $h \in X$ поставим в соответствие линейный оператор $B[h] \in \mathcal{L}(X, Y), B[h] x=B[h, x]$.

Для произвольных множеств $X$ и $Y$ и отображения $F: X \rightarrow Y$ через $F^{-1}: Y \rightarrow 2^{X}$ обозначается отображение, сопоставляющее каждому $y \in Y$ его полный прообраз при отображении $F$; если $F$ биективно, то считаем, что $F^{-1}: Y \rightarrow X$ - обычное (не многозначное) отображение. Если $X$ и $Y$ - линейные нормированные пространства, $\Lambda$ : $X \rightarrow Y$ - линейный оператор, то

$$
\left\|\Lambda^{-1}\right\|=\sup \left\{\rho_{X}\left(0, \Lambda^{-1} y\right) \mid y \in Y,\|y\|=1\right\}
$$

- "норма" отображения $\Lambda^{-1}$; в случае биективного $\Lambda$ это действительно операторная норма в пространстве $\mathcal{L}(Y, X)$.

Если $X_{i}, i=1, \ldots, s \quad(s \in \mathbb{N})$, и $Y$ - линейные нормированные пространства, $\Xi$ окрестность точки $\xi=\left(x^{1}, \ldots, x^{s}\right)$ в $\bigotimes_{i=1}^{s} X_{i}$, то для отображения $F: \Xi \rightarrow Y$ через $\partial_{i} F\left(x^{1}, \ldots, x^{s}\right)$ обозначается первая, а через $\partial_{i}^{2} F\left(x^{1}, \ldots, x^{s}\right)$ - вторая частная производная в точке $\xi$ по $i$-й переменной, $i=1, \ldots, s$.

Линейное подпространство $L$ банахова пространства $X$ называется топологически дополняемым, если оно замкнуто и имеет замкнутое прямое дополнение (называемое топологическим дополнением подпространства $L$ в $X$ ). Подпространство $L$ топологически дополняемо в $X$ тогда и только тогда, когда сушествует непрерьвньй $n p o е к т о р ~$ на $L$ в $X$, т.е. оператор $\Pi \in \mathcal{L}(X, X)$ такой, что $\Pi^{2}=\Pi, \operatorname{Im} \Pi=L$ (при этом дополнительный к Проектор $I_{X}-\Pi$ на топологическое дополнение $\operatorname{Ker} \Pi$ подпространства $L$ в $X$ также непрерывен).

\section{2. Теорема о представлении.}

Теорема 1. Пусть $\Sigma$ - топологическое, а $X$ и $Y$ - банаховы пространства, $\sigma_{0} \in \Sigma, V$ - окрестность точки $x_{*}$ в $X$. Пусть отображсение $F: \Sigma \times V \rightarrow Y$ удовлетворяет следуюшим условиям:

1) существуют оператор $A \in \mathcal{L}(X, Y)$ и симметричное отображение $B \in$ $\mathcal{L}^{2}(X, Y)$ такие, что для любого числа $\varepsilon>0$ найдутся окрестность $\mathcal{W}$ точки $\sigma_{0}$ в $\Sigma$ и число $\omega>0$ такие, что

$$
\begin{gathered}
\left\|F\left(\sigma, x_{*}+x^{1}\right)-F\left(\sigma, x_{*}+x^{2}\right)-A\left(x^{1}-x^{2}\right)-\frac{1}{2} B\left[x^{1}, x^{1}\right]+\frac{1}{2} B\left[x^{2}, x^{2}\right]\right\| \\
\leq \varepsilon\left(\left\|x^{1}\right\|+\left\|x^{2}\right\|\right)\left\|x^{1}-x^{2}\right\| \quad \forall \sigma \in \mathcal{W}, \quad \forall x^{1}, x^{2} \in B_{X}(0, \omega)
\end{gathered}
$$

(условие квадратичной аппроксимачии), причем $\operatorname{Im} A$ замкнуто и топологически дополняемо в $Y, R$ - непрерывный проектор на $\operatorname{Im} A$ в $Y, P=I_{Y}-R-$ дополнительный $к R$ проектор;

2) для некоторого конуса $K$ в $X$

$$
\sup \left\{\left\|(A+P B[h])^{-1}\right\| \mid h \in K,\|h\|=1\right\}<\infty .
$$

Тогда найдутся окрестности $\mathcal{U}$ точки $\sigma_{0}$ в $\Sigma$ и $U$ точки 0 в $X$ и отображение $\varphi: \mathcal{U} \times U \rightarrow X$ maкue, что

a) $\varphi(\mathcal{U} \times U) \subset V$; 
б) $F(\sigma, \varphi(\sigma, x))=F\left(\sigma, x_{*}\right)+A x+\frac{1}{2} P B[x, x] \forall \sigma \in \mathcal{U}, \forall x \in U \cap K$;

в) $\varphi(\sigma, 0)=x_{*} \quad \forall \sigma \in \mathcal{U}, u$ для некоторого числа $C>0$

$$
\begin{aligned}
&\left\|\varphi(\sigma, x)-\left(x_{*}+x\right)\right\| \leq C\left\|R\left(F\left(\sigma, x_{*}+x\right)-F\left(\sigma, x_{*}\right)-A x\right)\right\| \\
&\left.+\|x\|^{-1}\left\|P\left(F\left(\sigma, x_{*}+x\right)-F\left(\sigma, x_{*}\right)-\frac{1}{2} B[x, x]\right)\right\|\right) \\
& \forall \sigma \in \mathcal{U}, \quad \forall x \in U \backslash\{0\} .
\end{aligned}
$$

ДокАЗАТЕЛЬСТво. Фиксируем такое число $r>0$, что $B_{X}\left(x_{*}, 2 r\right) \subset V$. Тогда для каждых $\sigma \in \Sigma$ и $x \in B_{X}(0, r)$ корректно определено многозначное отображение

$$
\begin{gathered}
\Phi_{\sigma, x}: B_{X}(0, r) \rightarrow 2^{X}, \\
\Phi_{\sigma, x}(\xi)=\xi-(A+P B[x])^{-1}\left(F\left(\sigma, x_{*}+x+\xi\right)-F\left(\sigma, x_{*}\right)-A x-\frac{1}{2} P B[x, x]\right),
\end{gathered}
$$

для которого в силу (1) справедливо

$$
\Phi_{\sigma, x}(\xi) \neq \varnothing \quad \forall \sigma \in \Sigma, \quad \forall x \in\left(B_{X}(0, r) \cap K\right) \backslash\{0\}, \quad \forall \xi \in B_{X}(0, r) .
$$

Фиксируем число $\theta \in(0,1)$. Обозначим число, стоящее в левой части $(1)$, через $C^{\prime}$, $C^{\prime}>0$. Тогда в силу условия квадратичной аппроксимации найдутся окрестность $\mathcal{W}$ точки $\sigma_{0}$ в $\Sigma$ и числа $\omega \in(0, r]$ и $\gamma \in(0, r / \omega]$ такие, что $\forall \sigma \in \mathcal{W}, \forall x \in B_{X}(0, \omega)$, $\forall \xi^{1}, \xi^{2} \in B_{X}(0, \gamma\|x\|)$

$$
\begin{gathered}
\left\|R\left(F\left(\sigma, x_{*}+x+\xi^{1}\right)-F\left(\sigma, x_{*}+x+\xi^{2}\right)-A\left(\xi^{1}-\xi^{2}\right)\right)\right\| \leq \frac{\theta}{2 C^{\prime}}\left\|\xi^{1}-\xi^{2}\right\|, \\
\left\|P\left(F\left(\sigma, x_{*}+x+\xi^{1}\right)-F\left(\sigma, x_{*}+x+\xi^{2}\right)-B\left[x, \xi^{1}-\xi^{2}\right]\right)\right\| \leq \frac{\theta}{2 C^{\prime}}\|x\|\left\|\xi^{1}-\xi^{2}\right\| .
\end{gathered}
$$

Кроме того, вновь привлекая условие квадратичной апроксимации, получаем существование окрестностей $\mathcal{U} \subset \mathcal{W}$ точки $\sigma_{0}$ в $\Sigma$ и $U \subset B_{X}(0, \omega)$ точки 0 в $X$ таких, что $\forall \sigma \in \mathcal{U}, \forall x \in U \backslash\{0\}$

$$
\begin{aligned}
\left\|R\left(F\left(\sigma, x_{*}+x\right)-F\left(\sigma, x_{*}\right)-A x\right)\right\| & <\frac{1-\theta}{2 C^{\prime}} \gamma\|x\|, \\
\left\|P\left(F\left(\sigma, x_{*}+x\right)-F\left(\sigma, x_{*}\right)-\frac{1}{2} B[x, x]\right)\right\| & <\frac{1-\theta}{2 C^{\prime}} \gamma\|x\|^{2} .
\end{aligned}
$$

Положим $r(x)=\gamma\|x\|, x \in B_{X}(0, \omega)$. В силу $(1),(3),(4)$ для хаусдорфова расстояния $\aleph_{X}(\cdot, \cdot)$ в пространстве $X$ имеем: $\forall \sigma \in \mathcal{U}, \forall x \in(U \cap K) \backslash\{0\}, \forall \xi^{1}, \xi^{2} \in B_{X}(0, r(x))$

$$
\begin{aligned}
\aleph_{X}( & \left.\Phi_{\sigma, x}\left(\xi^{1}\right), \Phi_{\sigma, x}\left(\xi^{2}\right)\right)=\inf \left\{\left\|x^{1}-x^{2}\right\| \mid x^{i} \in \Phi_{\sigma, x}\left(\xi^{i}\right), i=1,2\right\} \\
= & \inf \left\{\|\xi\| \mid \xi \in X,\left(A+P B\left[\|x\|^{-1} x\right]\right) \xi=\left(A+P B\left[\|x\|^{-1} x\right]\right)\left(\xi^{1}-\xi^{2}\right)\right. \\
& \quad-R\left(F\left(\sigma, x_{*}+x+\xi^{1}\right)-F\left(\sigma, x_{*}+x+\xi^{2}\right)\right) \\
& \left.\quad-\|x\|^{-1} P\left(F\left(\sigma, x_{*}+x+\xi^{1}\right)-F\left(\sigma, x_{*}+x+\xi^{2}\right)\right)\right\} \\
\leq & C^{\prime}\left(\left\|R\left(F\left(\sigma, x_{*}+x+\xi^{1}\right)-F\left(\sigma, x_{*}+x+\xi^{2}\right)-A\left(\xi^{1}-\xi^{2}\right)\right)\right\|\right. \\
+ & \left.\|x\|^{-1}\left\|P\left(F\left(\sigma, x_{*}+x+\xi^{1}\right)-F\left(\sigma, x_{*}+x+\xi^{2}\right)-B\left[x, \xi^{1}-\xi^{2}\right]\right)\right\|\right) \leq \theta\left\|\xi^{1}-\xi^{2}\right\| .
\end{aligned}
$$


Аналогично, в силу (1), (5), (6) получаем: $\forall \sigma \in \mathcal{U}, \forall x \in(U \cap K) \backslash\{0\}$

$$
\begin{aligned}
\rho_{X}\left(0, \Phi_{\sigma, x}(0)\right)= & \inf \left\{\|\xi\| \mid \xi \in \Phi_{\sigma, x}(0)\right\}=\inf \left\{\|\xi\| \mid \xi \in X,\left(A+P B\left[\|x\|^{-1} x\right]\right) \xi\right. \\
= & R\left(F\left(\sigma, x_{*}+x\right)-F\left(\sigma, x_{*}\right)-A x\right) \\
& \left.+\|x\|^{-1} P\left(F\left(\sigma, x_{*}+x\right)-F\left(\sigma, x_{*}\right)-\frac{1}{2} B[x, x]\right)\right\} \\
\leq & C^{\prime}\left(\left\|R\left(F\left(\sigma, x_{*}+x\right)-F\left(\sigma, x_{*}\right)-A x\right)\right\|\right. \\
& \left.+\|x\|^{-1}\left\|P\left(F\left(\sigma, x_{*}+x\right)-F\left(\sigma, x_{*}\right)-\frac{1}{2} B[x, x]\right)\right\|\right)<(1-\theta) r(x) .
\end{aligned}
$$

Соотношения $(2),(7),(8)$ в совокупности означают, что для каждых $\sigma \in \mathcal{U}$ и $x \in$ $(U \cap K) \backslash\{0\}$ отображение $\Phi_{\sigma, x}$ в шаре $B_{X}(0, r(x))$ удовлетворяет всем условиям принципа многозначных сжимающих отображений $[4$, c. 42$]$, т.е. найдется элемент $\xi(\sigma, x) \in$ $B_{X}(0, r(x))$ такой, что

$$
\xi(\sigma, x) \in \Phi_{\sigma, x}(\xi(\sigma, x))
$$

причем

$$
\|\xi(\sigma, x)\| \leq \frac{2}{1-\theta} \rho_{X}\left(0, \Phi_{\sigma, x}(0)\right) .
$$

Положим $\xi(\sigma, 0)=0 \forall \sigma \in \mathcal{U}$. Тогда в силу $(9),(10)$ и промежуточного неравенствав $(8)$ отображение

$$
\varphi: \mathcal{U} \times U \rightarrow X, \quad \varphi(\sigma, x)= \begin{cases}x_{*}+x+\xi(\sigma, x), & \text { если } x \in U \cap K, \\ x_{*}+x, & \text { если } x \in U \backslash K,\end{cases}
$$

является искомым (в качестве константы в в) следует взять $\left.C=2 C^{\prime} /(1-\theta)\right)$. Теорема доказана.

ЗАмЕчАниЕ 1. Условие квадратичной аппроксимации включает в себя и так называемое условие линейной аппроксимации: существует оператор $A \in \mathcal{L}(X, Y)$ такой, что для любого числа $\varepsilon>0$ найдутся окрестность $\mathcal{W}$ точки $\sigma_{0}$ в $\Sigma$ и число $\omega>0$ такие, что

$\left\|F\left(\sigma, x_{*}+x^{1}\right)-F\left(\sigma, x_{*}+x^{2}\right)-A\left(x^{1}-x^{2}\right)\right\| \leq \varepsilon\left\|x^{1}-x^{2}\right\| \quad \forall \sigma \in \mathcal{W}, \quad \forall x^{1}, x^{2} \in B_{X}(0, \omega)$

(см. [1, с. 161]). Последнее же предполагает строгую дифференцируемость отображения $x \rightarrow F\left(\sigma_{0}, x\right): V \rightarrow Y$ в точке $x_{*}$, причем по необходимости $A=\partial_{2} F\left(\sigma_{0}, x_{*}\right)$. Наоборот, если существует такая окрестность $\mathcal{V}$ точки $\sigma_{0}$ в $\Sigma$, что отображение $F$ дифференцируемо по $\Phi$ реше по второй переменной на $\mathcal{V} \times V$, причем отображение $\partial_{2} F$ : $\mathcal{V} \times V \rightarrow \mathcal{L}(X, Y)$ непрерывно в точке $\left(\sigma_{0}, x_{*}\right)$, то условие линейной апшроксимации выполнено с указанным оператором $A$ (это следует из теоремы о среднем).

ЗАмЕчАниЕ 2. Правая часть представления в б) содержит $\sigma$, а не только $\sigma_{0}$. Усилить данньй результат, избавившись от указанной зависимости в представлении, в общем случае нельзя, что иллюстрируется простейшим примером отображения $F$ : $\mathbb{R} \times \mathbb{R} \rightarrow \mathbb{R}, F(\sigma, x)=\sigma+x^{2}$ в точке $(0,0)$ (здесь можно взять $\left.K=\mathbb{R}\right)$. 
ЗАмЕчАнИЕ 3. Из утверждения в) теоремы 1 и условия квадратичной аппроксимации вытекает, что отображение $x \rightarrow \varphi\left(\sigma_{0}, x\right): U \rightarrow X$ можно считать дифференцируемым по Фреше в точке 0, причем $\partial_{2} \varphi\left(\sigma_{0}, 0\right)=I_{X}$.

Ниже обсуждаются некоторые приложения теоремы 1.

3. Теоремы о существовании неявной функции. В [3] доказана следующая теорема о существовании неявной функции.

Теорема 2*. Пусть в условиях теоремы $1 F\left(\sigma_{0}, x_{*}\right)=0$, а вместо 2) выполнено следующее:

$\left.2^{*}\right)$ существует әлемент $h_{*} \in \operatorname{Ker} A$ такой, что $B\left[h_{*}, h_{*}\right] \in \operatorname{Im} A, \operatorname{Im}(A+$ $\left.P B\left[h_{*}\right]\right)=Y$;

3) отобрахсение $\sigma \rightarrow F\left(\sigma, x_{*}\right): \Sigma \rightarrow Y$ непрерывно в точке $\sigma_{0}$ (условие непрерывности).

Тогда найдутся окрестность $\mathcal{U}$ точки $\sigma_{0}$ в $\Sigma$ и отображсение $\chi: \mathcal{U} \rightarrow X$ такие, чmo:

a) $\chi(\mathcal{U}) \subset V$;

б) $F(\sigma, \chi(\sigma))=0 \forall \sigma \in \mathcal{U}$;

в) для некоторого числа $C>0$

$$
\left\|\chi(\sigma)-x_{*}\right\| \leq C\left(\left\|R F\left(\sigma, x_{*}\right)\right\|+\left\|P F\left(\sigma, x_{*}\right)\right\|^{1 / 2}\right) \quad \forall \sigma \in \mathcal{U}
$$

В регулярном случае, т.е. когда $\operatorname{Im} A=Y$, условие $2^{*}$ ) теоремы $2^{*}$ вьполнено тривиальньм образом для любого отображения $B \in \mathcal{L}^{2}(X, Y)$, поскольку при этом $P=0$, и в качестве $h_{*}$ можно взять любой элемент из $\operatorname{Ker} A$, например, $h_{*}=0$. Поэтому с учетом замечания 1 теорема $2^{*}$ является обобщением стандартной теоремы о существовании неявной функции из $[1$, с. 161$]$ (см. также замечание 4 ниже). Однако, такое обобщение не может считаться исчерпьвающим, например потому, что теорема 2 * в нерегулярном случае принщипиально неприменима при $X=Y=\mathbb{R}^{n}, n \in \mathbb{N}$. Привлекая дополнительно теорему 1 , приходим к следующему результату.

Теорема 2. Пусть в условиях теоремы $1 F\left(\sigma_{0}, x_{*}\right)=0$, причем выполнено условие 3) теоремы $2^{*}$. Введем множество

$$
\widetilde{\Sigma}=\left\{\sigma \in \Sigma \mid \exists x \in K:-F\left(\sigma, x_{*}\right)=A x+\frac{1}{2} P B[x, x]\right\} .
$$

Тогда найдутся окрестность $\mathcal{U}$ точки $\sigma_{0}$ в $\Sigma$ и отображсние $\chi: \mathcal{U} \rightarrow X$ такие, что:

a) $\chi(\mathcal{U}) \subset V$;

б) $F(\sigma, \chi(\sigma))=0 \forall \sigma \in \mathcal{U} \cap \widetilde{\Sigma}$;

в) для некоторого числа $C>0$

$$
\left\|\chi(\sigma)-x_{*}\right\| \leq C\left(\left\|R F\left(\sigma, x_{*}\right)\right\|+\left\|P F\left(\sigma, x_{*}\right)\right\|^{1 / 2}\right) \quad \forall \sigma \in \mathcal{U}
$$


ДокАЗАТЕЛЬСтво. Предположим сначала, что существует такое число $\beta>0$, что

$$
\left\{x \in K_{\beta} \mid A x=0, P B[x, x]=0\right\}=\{0\},
$$

где

$$
K_{\beta}=\bigcup_{x \in K} B_{X}(x, \beta\|x\|) .
$$

Но тогда в силу леммы 3 в [2] найдется такое число $C^{\prime}>0$, что

$$
\|x\| \leq C^{\prime}\left(\|A x\|+\left\|\frac{1}{2} P B[x, x]\right\|^{1 / 2}\right) \quad \forall x \in K .
$$

Поэтому согласно $(11)$ для каждого $\sigma \in \widetilde{\Sigma}$ найдется такой элемент $x(\sigma) \in K$, что

$$
\begin{gathered}
A x(\sigma)+\frac{1}{2} P B[x(\sigma), x(\sigma)]=-F\left(\sigma, x_{*}\right), \\
\|x(\sigma)\| \leq C^{\prime}\left(\left\|R F\left(\sigma, x_{*}\right)\right\|+\left\|P F\left(\sigma, x_{*}\right)\right\|^{1 / 2}\right) .
\end{gathered}
$$

Пусть окрестности $\mathcal{U}, U$ и отображение $\varphi$ определены в соответствии с теоремой 1 . В силу (14) и условия непрерывности можем при необходимости сузить окрестность $\mathcal{U}$ так, чтобы вьполнялось $x(\sigma) \in U \forall \sigma \in \mathcal{U}$. Тогда согласно (13) искомым будет отображение

$$
\chi: \mathcal{U} \rightarrow X, \quad \chi(\sigma)= \begin{cases}\varphi(\sigma, x(\sigma)), & \text { если } \sigma \in \mathcal{U} \cap \widetilde{\Sigma}, \\ x_{*}, & \text { если } x \in \mathcal{U} \backslash \widetilde{\Sigma}\end{cases}
$$

(для обоснования утверждения в) нужно привлечь условие квадратичной аппроксимациии (14) и, возможно, еще сузить окрестность $\mathcal{U})$.

Пусть теперь условие (12) не выполнено ни для какого числа $\beta>0$. Используя теорему о малом возмущении сюръективного линейного оператора [5, с. 26], несложно убедиться, что при этом выполнены все условия теоремы $2^{*}$, что и дает требуемьй результат. Теорема доказана.

Вообще говоря, расширить множество $\widetilde{\Sigma}$ (например, положив $\widetilde{\Sigma}=\Sigma$ ) в утверждении теоремы 2 нельзя (см. замечание 2).

Условие (1) в теоремах 1 и 2 (как и условие $2^{*}$ ) в теореме $2^{*}$ ) играет роль ослабленного условия регулярности. Условия такого рода подробно обсуждаются в [2], [6]. Здесь отметим лишь, что если размерность $X$ конечна и нечетна, $\operatorname{dim} Y \geq 2$, то в нерегулярном случае вьполнение $(1)$ при $K=X$ паталогично.

Важно отметить, что теорема $2^{*}$ содержится в теореме 2 . Действительно, в условиях теоремы $2^{*}$, вновь привлекая теорему о малом возмущении сюръективного линейного оператора, выберем число $\beta>0$ такое, что

$$
\sup \left\{\left\|(A+P B[h])^{-1}\right\| \mid h \in B_{X}\left(h_{*}, \beta\right)\right\}<\infty,
$$

и положим $K=$ cone $B_{X}\left(h_{*}, \beta\right)$. Тогда, применяя к отображению

$$
\Phi: X \rightarrow Y, \quad \Phi(x)=A x+\frac{1}{2} P B[x, x],
$$

в точке $h_{*}$ стандартную теорему о существовании неявной функции $[1$, с. 161$]$ (точнее, теорему о накрьвании), имеем: найдется такая окрестность $W$ точки 0 в $Y$, что

$$
\Phi^{-1}(y) \cap K \neq \varnothing \quad \forall y \in W .
$$

Отсюда, используя условие непрерьвности, получаем, что вводимое в соответствии с (11) множество $\widetilde{\Sigma}$ является окрестностью точки $\sigma_{0}$ в $\Sigma$, что и дает требуемое. 
ЗАмЕчАниЕ 4. Если отображение $x \rightarrow F\left(\sigma_{0}, x\right): V \rightarrow Y$ дважды дифференцируемо по $\Phi$ реше в точке $x_{*}$, то по необходимости в условии квадратичной аппроксимации $B=\partial_{2}^{2} F\left(\sigma_{0}, x_{*}\right)$. Отметим, что в приведенных теоремах условие квадратичной апшроксимации можно несколько ослабить: достаточно предполагать, что выполнено условие линейной аппроксимации (см. замечание 1) и существует симметричное отображение $B \in \mathcal{L}^{2}(X, Y)$ такое, что для любого числа $\varepsilon>0$ найдутся окрестность $\mathcal{W}$ точки $\sigma_{0}$ в $\Sigma$ и число $\omega>0$ такие, что

$$
\begin{gathered}
\left\|P\left(F\left(\sigma, x_{*}+x^{1}\right)-F\left(\sigma, x_{*}+x^{2}\right)-\frac{1}{2} B\left[x^{1}, x^{1}\right]+\frac{1}{2} B\left[x^{2}, x^{2}\right]\right)\right\| \\
\leq \varepsilon\left(\left\|x^{1}\right\|+\left\|x^{2}\right\|\right)\left\|x^{1}-x^{2}\right\| \quad \forall \sigma \in \mathcal{W}, \quad \forall x^{1}, x^{2} \in B_{X}(0, \omega) .
\end{gathered}
$$

Например, не зависящее от параметра отображение $F: \mathbb{R}^{2} \rightarrow \mathbb{R}^{2}, F(x)=$ $\left(x_{1}+\left|x_{1}\right|^{3 / 2}, 0\right)$, в точке 0 удовлетворяет ослабленному условию квадратичной апшроксимации, но не имеет второй производной и не удовлетворяет условию квадратичной аппроксимации. В соответствующей формулировке теорема $2 *$ содержит в себе стандартную теорему о существовании неявной функции из [1, с. 161] без всяких оговорок.

Кроме того, нужно иметь в виду, что в отличие от условия линейной апроксимации условие квадратичной аппроксимации - не просто условие гладкости. Никакие требования гладкости на отображение $F$, вообще говоря, не гарантируют вьполнение условия квадратичной аппроксимации (даже ослабленного), что иллюстрируется примером отображения $F: \mathbb{R} \times \mathbb{R} \rightarrow \mathbb{R}, F(\sigma, x)=\sigma x$, в точке $(0,0)$.

Можно указать следующие условия, достаточные для вьполнения ослабленного условия квадратичной аппроксимации: существует такая окрестность $\mathcal{V}$ точки $\sigma_{0}$ в $\Sigma$, что отображение $F$ дважды дифференцируемо по $\Phi$ реше по второй переменной на $\mathcal{V} \times V$, причем отображения $\partial_{2} F: \mathcal{V} \times V \rightarrow \mathcal{L}(X, Y)$ и $\partial_{2}^{2} F: \mathcal{V} \times V \rightarrow \mathcal{L}^{2}(X, Y)$ непрерьвны в точке $\left(\sigma_{0}, x_{*}\right)$ и

$$
P \partial_{2} F\left(\sigma, x_{*}\right)=0 \quad \forall \sigma \in \mathcal{V}
$$

(это следует из теоремы о среднем). В теоремах $2^{*}$ и 2 вместо (15) достаточно предполагать вьполненным более слабое условие: для любого числа $\varepsilon>0$ найдется окрестность $\mathcal{W} \subset \mathcal{V}$ точки $\sigma_{0}$ в $\Sigma$ такая, что

$$
\left\|P \partial_{2} F\left(\sigma, x_{*}\right)\right\| \leq \varepsilon\left(\left\|R F\left(\sigma, x_{*}\right)\right\|+\left\|P F\left(\sigma, x_{*}\right)\right\|^{1 / 2}\right) \quad \forall \sigma \in \mathcal{W}
$$

(см. [2]). Заметим, что такие условия не гарантируют выполнения условия квадратичной аппроксимации (даже ослабленного); простейшим примером служит отображение $F: \mathbb{R} \times \mathbb{R} \rightarrow \mathbb{R}, F(\sigma, x)=\sigma+\sigma x$, в точке $(0,0)$.

В теореме 1 указанная замена условий не проходит даже при выполнении условия непрерьвности, что иллюстрируется примером отображения $F: \mathbb{R} \times \mathbb{R}^{2} \rightarrow \mathbb{R}^{2}, F(\sigma, x)=$ $\left(x_{1}, \sigma+\sigma x_{1}+x_{2}^{2}\right)$, в точке $(0,0)$.

Наконец, приведем еще одни условия (с пониженными требованиями гладкости), достаточные для вьполнения условия квадратичной аппоксимации: существует такая окрестность $\mathcal{V}$ точки $\sigma_{0}$ в $\Sigma$, что отображение $F$ дифференцируемо по Фреше по второй переменной на $\mathcal{V} \times V$ и дважды дифференцируемо по Фреше по второй переменной в точке $\left(\sigma_{0}, x_{*}\right)$, причем

$$
\partial_{2} F(\sigma, x)=\partial_{2} F\left(\sigma_{0}, x\right) \quad \forall \sigma \in \mathcal{V}, \forall x \in V
$$


(это следует из теоремы о среднем). В частности, если отображение $F: V \rightarrow Y$ не зависит от параметра, то для вьполнения условия квадратичной аппоксимации достаточно двукратной дифференцируемости $F$ по $\Phi$ реше в точке $x_{*}$.

В связи с замечанием 4 еще раз подчеркнем, что ни одна из теорем 1 и 2 не содержит в себе другую полностью.

4. Другие приложения. Оговорим еще некоторые понятия и обозначения [3], [5], [6]. Пусть $X$ и $Y$ - банаховы пространства, $V$ - окрестность точки $x_{*}$ в $X$, отображение $F: V \rightarrow Y$ дважды дифференцируемо по $\Phi$ реше в точке $x_{*}$. Полагая, что $P-$ проектор на некоторое прямое дополнение $\operatorname{Im} F^{\prime}\left(x_{*}\right)$ в $Y$, введем семейство линейных операторов

$$
\Psi_{2}(P, h): X \rightarrow Y, \quad \Psi_{2}(P, h)=F^{\prime}\left(x_{*}\right)+P F^{\prime \prime}\left(x_{*}\right)[h], \quad h \in X,
$$

и конус

$$
T_{2}=\left\{h \in \operatorname{Ker} F^{\prime}\left(x_{*}\right) \mid F^{\prime \prime}\left(x_{*}\right)[h, h] \in \operatorname{Im} F^{\prime}\left(x_{*}\right)\right\} .
$$

ОПРЕДЕЛЕНИЕ 1. Отображение $F$ назьвается 2-регулярным в точке $x_{*}$ на әлементе $h \in X$, если $\operatorname{Im} \Psi_{2}(P, h)=Y$.

ОПРЕДЕЛЕНИЕ 2. Отображение $F$ называется 2-регулярным в точке $x_{*}$ на множестве $H \subset X$, если оно 2-регулярно в этой точке на любом элементе $h \in X$.

ОПРЕДЕЛЕНИЕ 3 . Отображение $F$ называется 2-регулярным в точке $x_{*}$, если оно 2 -регулярно в этой точке на множестве $T_{2} \backslash\{0\}$.

Подчеркнем, что введенные условия 2-регулярности на самом деле инвариантны по отоношению к выбору прямого дополнения подпространства $\operatorname{Im} F^{\prime}\left(x_{*}\right)$ в $Y$, т.е. к выбору проектора $P$. Очевидно, если отображение $F$ регулярно в точке $x_{*}$, то оно 2 -регулярно в этой точке на множестве $X$.

Привлекая замечания 3 и 4, получаем такое следствие теоремы 1 (в [2] этот результат о представлении нелинейного отображения доказан непосредственно).

Теорема 3. Пусть $X$ и $Y$ - банаховы пространства, $V$ - окрестность точ$\kappa и x_{*}$ в $X$. Пусть отображение $F: V \rightarrow Y$ дважды дифференцируемо по Фреше в точке $x_{*}, \operatorname{Im} F^{\prime}\left(x_{*}\right)$ замкнуто и топологически дополняемо в $Y, R$ - непрерывный проектор на $\operatorname{Im} F^{\prime}\left(x_{*}\right)$ в $Y, P=I_{Y}-R$ - дополнительный $\kappa R$ проектор, причем для некоторго конуса $K$ в $X$

$$
\sup \left\{\left\|\left(\Psi_{2}(P, h)\right)^{-1}\right\| \mid h \in K,\|h\|=1\right\}<\infty .
$$

Тогда найдутся окрестность $U$ точки 0 в $X$ и дифференцируемое по Фреше в точке 0 отобрахсение $\varphi: U \rightarrow X$ такие, что

a) $\varphi(U) \subset V$;

б) $F(\varphi(x))=F\left(x_{*}\right)+F^{\prime}\left(x_{*}\right) x+\frac{1}{2} P F^{\prime \prime}\left(x_{*}\right)[x, x] \forall x \in U \cap K$;

в) $\varphi(0)=x_{*}, \varphi^{\prime}(0)=I_{X}$.

В регулярном случае теорема 3 дает некоторьй результат о линеаризации нелинейного отображения. Отметим, что “выпрямляющее” отображение $\varphi$ из теоремы 3 не удовлетворяет, вообще говоря, условиям стандартных теорем об обратном отображении, несмотря на существование и биективность производной $\varphi^{\prime}(0)$. Дело в том, что $\varphi(\cdot)$ 
не обладает, вообще говоря, строгой дифференцируемостью в точке 0 (в частности, в окрестности этой точки не утверждается даже непрерывность $\varphi(\cdot))$. Таким образом, $\varphi(\cdot)$ нельзя считать “заменой переменных" (об условиях, обеспечивающих локальную биективность и гладкость $\varphi(\cdot)$, т.е. об обобщении классической леммы Морса, см. [2]). Тем не менее, результат теоремы 3 достаточен для ряда важных приложений.

Например, теорема 3 содержит в себе обобщенную теорему Люстерника о касательном конусе [3], [5, с. 157]. Будем рассматривать множество уровня

$$
M=\left\{x \in V \mid F(x)=F\left(x_{*}\right)\right\}
$$

отображения $F$, содержащее точку $x_{*}$.

ТЕорема 4. Пусть выполнены условия теоремы 3, кроме (16).

Тогда для множества уровня $M$ справедливо:

a) $T_{x_{*}} M \subset T_{2}$

б) если отобрахсение $F$ 2-регулярно в точке $x_{*}$ на әлементе $h \in T_{2}$, то $h \in$ $T_{x_{*}} M$.

$B$ частности, если отображсене $F$ 2-регулярно в точке $x_{*}$, то $T_{x_{*}} M=T_{2}$.

Действительно, в условиях п.б) имеем:

$$
F(\varphi(t h))=F\left(x_{*}\right)+t F^{\prime}\left(x_{*}\right) h+\frac{t^{2}}{2} P F^{\prime \prime}\left(x_{*}\right)[h, h]=F\left(x_{*}\right) \quad \forall t \in(-\varepsilon, \varepsilon),
$$

где число $\varepsilon>0$ определяется из условия $t h \in U \forall t \in(-\varepsilon, \varepsilon)$, а окрестность $U$ и отображение $\varphi$ взяты из теоремы 3 , применяемой к конусу $K=\operatorname{lin} h$. При этом

$$
\left\|\varphi(t h)-\left(x_{*}+t h\right)\right\|=o(t) .
$$

Отметим такое обобщение стандартного результата о накрьвании, содержашееся в теореме $2^{*}$ (с учетом замечания 4 ).

ТЕОРема 5. Пусть в условиях теоремы 3 вместо (16) выполнено следующее: отображение $F$ 2-регулярно в точке $x_{*}$ на некотором элементе $h_{*} \in T_{2}$.

Тогда найдутся окрестность $W$ точки $F\left(x_{*}\right)$ в $Y$ и число $C>0$ такие, что

$$
\rho_{X}\left(x_{*}, F^{-1}(y)\right) \leq C\left(\left\|R\left(y-F\left(x_{*}\right)\right)\right\|+\left\|P\left(y-F\left(x_{*}\right)\right)\right\|^{1 / 2}\right) \quad \forall y \in W .
$$

Если провести аналогию с регулярным случаем, то можно предположить справедливость такого следствия теоремы $2^{*}$ : равенство $T_{x_{*}} M=T_{2}$ в теореме 4 имеет место при вьполнении условия 2-регулярности отображения $F$ в точке $x_{*}$ хотя бы на одном элементе из конуса $T_{2}$. Однако, последнее неверно. Точнее, если обозначить $\bar{T}_{2}=\{\xi \in$ $\left.T_{2} \mid \operatorname{Im} \Psi_{2}(P, \xi)=Y\right\}$, то из теоремы 4 и замкнутости касательного конуса к любому множеству в любой точке $[4, \mathrm{c.41}]$ вытекает включение $\mathrm{cl} \bar{T}_{2} \subset T_{x_{*}} M$; включение же $T_{2} \backslash \operatorname{cl} \bar{T}_{2} \subset T_{x_{*}} M$ может не иметь места, даже если $\bar{T}_{2} \neq \varnothing$. Например, для отображения $F: \mathbb{R}^{3} \rightarrow \mathbb{R}^{2}, F(x)=\left(x_{1}^{2}+x_{2}^{2}-x_{3}^{2}, x_{1}\left(x_{1}-x_{3}-x_{3}^{2}\right)\right)$, в точке 0 имеем: $(0, \pm 1,1) \in \bar{T}_{2}$, $(1,0,1) \in T_{2}$, но, как нетрудно убедиться, последний вектор не является касательным к множеству $M$.

В заключение этого раздела отметим недавно опубликованный вариант обобщенной теоремы о накрьвании [7], основанный на совершенно иных идеях. 
5. О гладких уточнениях теорем о неявной функции. Оценки в) в теоремах $2^{*}, 2$ гарантируют непрерьвность неявной функции $\chi$ в точке $\sigma_{0}$. Никакие дополнительные требования гладкости на отображение $F$ не позволяют в условиях этих теорем гарантировать сушествование гладких ветвей решений (при естественной параметризации; напомним, что в условиях теоремы 2 такая “ветвь" вообще определена на множестве, которое может не содержать окрестности точки $\sigma_{0}$ в $\Sigma$ ). Примером может служить бесконечно гладкое по совокупности переменных отображение $F: \mathbb{R} \times \mathbb{R}^{2} \rightarrow \mathbb{R}$, $F(\sigma, x)=\sigma-x_{1} x_{2}$, в точке $(0,0)$. Действительно, здесь вьполнены все условия теоремы $2^{*}$, но если предположить существование окрестности $\mathcal{U}$ точки 0 в $\mathbb{R}$ и дифференцируемого хотя бы в самой точке 0 отображения $\chi: \mathcal{U} \rightarrow \mathbb{R}^{2}$ таких, что

$$
\begin{aligned}
\chi_{1}(\sigma) \chi_{2}(\sigma) & =\sigma \quad \forall \sigma \in \mathcal{U}, \\
\chi(0) & =0
\end{aligned}
$$

то, дифференцируя левую и правую части равенства (17) по $\sigma$ в точке 0, получим

$$
\dot{\chi}_{1}(0) \chi_{2}(0)+\chi_{1}(0) \dot{\chi}_{2}(0)=1 \text {. }
$$

Последнее равенство противоречит (18).

Обратимся к другому вопросу: можно ли за счет повьшенных требований гладкости на отображение $F$ снять в теоремах $2^{*}, 2$ весьма обременительное условие квадратичной аппорксимации, положив $A=\partial_{2} F\left(\sigma_{0}, x_{*}\right), B=\partial_{2}^{2} F\left(\sigma_{0}, x_{*}\right)$ (см. замечание 4)? В случае теоремы $2^{*}$ положительньй ответ на этот вопрос может быть получен с помощью известного приема, позволяющего вьводить теоремы о неявной функции из соответствующих теорем об обратном отображении (на возможность применения данного приема в рассматриваемой ситуации автору указал А. В. Арутюнов).

Теорема 6. Пусть $\Sigma, X u Y$ - банаховы пространства, $\mathcal{V}$ - окрестность точ$\kappa и \sigma_{0}$ в $\Sigma, V-$ точки $x_{*}$ в $X$. Пусть отображсение $F: \mathcal{V} \times V \rightarrow Y$ дважды дифферениируемо по Фреше в точке $\left(\sigma_{0}, x_{*}\right), \operatorname{Im} \partial_{2} F\left(\sigma_{0}, x_{*}\right)$ замкнуто и топологически дополняемо в $Y, R$-некоторый непрерывный проектор на $\operatorname{Im} \partial_{2} F\left(\sigma_{0}, x_{*}\right)$ в $Y, P=I_{Y}-R$ - дополнительный $\kappa R$ проектор, причем существует әлемент $h_{*} \in \operatorname{Ker} \partial_{2} F\left(\sigma_{0}, x_{*}\right)$ такой, что $\partial_{2}^{2} F\left(\sigma_{0}, x_{*}\right)\left[h_{*}, h_{*}\right] \in \operatorname{Im} \partial_{2} F\left(\sigma_{0}, x_{*}\right)$ и отображсение $x \rightarrow F\left(\sigma_{0}, x\right): V \rightarrow Y$ 2-регулярно в точке $x_{*}$ на әлементе $h_{*}$. Пусть $F\left(\sigma_{0}, x_{*}\right)=0$.

Тогда найдутся окрестность $\mathcal{U} \subset \mathcal{V}$ точки $\sigma_{0}$ в $\Sigma$ и отображсние $\chi: \mathcal{U} \rightarrow X$ такие, что:

a) $\chi(\mathcal{U}) \subset V$;

б) $F(\sigma, \chi(\sigma))=0 \forall \sigma \in \mathcal{U}$;

в) для некоторого числа $C>0$

$$
\left\|\chi(\sigma)-x_{*}\right\| \leq C\left(\left\|\sigma-\sigma_{0}\right\|+\left\|P \partial_{1} F\left(\sigma_{0}, x_{*}\right)\left(\sigma-\sigma_{0}\right)\right\|^{1 / 2}\right) \quad \forall \sigma \in \mathcal{U}
$$


ДокАЗАТЕЛЬСтво. Введем открытое множество $\mathcal{O}=\mathcal{V} \times V$ в пространстве $\Sigma \times X$ и отображение

$$
\Phi: \mathcal{O} \rightarrow E, \quad \Phi(z)=(\sigma, F(\sigma, x)), \quad z=(\sigma, x),
$$

где $E=\Sigma \times Y$. Непосредственно проверяется, что в сделанных предположениях это отображение удовлетворяет всем условиям теоремы 5 в точке $z_{*}=\left(\sigma_{0}, x_{*}\right)$. В частности, $\Phi 2$-регулярно в точке $z_{*}$ на элементе $\zeta_{*}=\left(0, h_{*}\right) \in \operatorname{Ker} \Phi^{\prime}\left(z_{*}\right)=\{0\} \times \operatorname{Ker} \partial_{2} F\left(\sigma_{0}, x_{*}\right)$, причем $\Phi^{\prime \prime}\left(z_{*}\right)\left[\zeta_{*}, \zeta_{*}\right] \in \operatorname{Im} \Phi^{\prime}\left(z_{*}\right)$. Отметим, что в качестве непрерывного проектора на подпространство $\operatorname{Im} \Phi^{\prime}\left(z_{*}\right)$ в $E$ можно взять, например, оператор $T$, определяемьй формулой

$$
T e=\left(\mu, R y+P \partial_{1} F\left(\sigma_{0}, x_{*}\right) \mu\right), \quad e=(\mu, y) \in E .
$$

В силу теоремы 5 найдутся окрестности $\mathcal{U} \subset \mathcal{V}$ точки $\sigma_{0}$ в $\Sigma$ и $W$ точки 0 в $Y$ и отображение $\psi: \mathcal{U} \times W \rightarrow X$ такие, что:

$$
\begin{gathered}
\psi(\mathcal{U} \times W) \subset V, \\
F(\sigma, \psi(\sigma, y))=y \quad \forall \sigma \in \mathcal{U}, \quad \forall y \in W,
\end{gathered}
$$

причем для некоторого числа $C^{\prime}>0$

$$
\begin{aligned}
& \left\|\psi(\sigma, y)-z_{*}\right\| \leq C^{\prime}\left(\left\|\left(\begin{array}{c}
\sigma-\sigma_{0} \\
R y+P \partial_{1} F\left(\sigma_{0}, x_{*}\right)\left(\sigma-\sigma_{0}\right)
\end{array}\right)\right\|\right. \\
& \left.\quad+\left\|\left(\begin{array}{c}
0 \\
P\left(y-\partial_{1} F\left(\sigma_{0}, x_{*}\right)\left(\sigma-\sigma_{0}\right)\right)
\end{array}\right)\right\|^{1 / 2}\right) \quad \forall \sigma \in \mathcal{U}, \quad \forall y \in W .
\end{aligned}
$$

Тогда искомое отображение $\chi$ определяется формулой

$$
\chi(\sigma)=\psi(\sigma, 0), \quad \sigma \in \mathcal{U}
$$

(оценка в) вьполняется при $C=C^{\prime} \max \left\{1,\left\|P \partial_{1} F\left(\sigma_{0}, x_{*}\right)\right\|\right\}$ ). Теорема доказана.

Оценка в) в теореме $2^{*}$, вообще говоря, сильнее оценки в) в теореме 6 . Тем не менее, и последняя оценка вполне содержательна.

Что же касается общей теоремы 2 , то в ней никакие дополнительные требования гладкости на отображение $F$ не позволяют отказаться от условий типа квадратичной аппроксимации. Рассмотрим, например, бесконечно гладкое по совокупности переменных отображение

$$
F: \mathbb{R} \times \mathbb{R}^{2} \rightarrow \mathbb{R}^{2}, \quad F(\sigma, x)=\left(\sigma+x_{1}+x_{2}, \frac{3}{8} \sigma\left(x_{1}+x_{2}\right)+x_{1} x_{2}\right),
$$

в точке $(0,0)$. Зафиксируем произвольное число $\nu \in(0,1]$ и введем конус

$$
\begin{array}{r}
K=\left\{x \in \mathbb{R}^{2} \mid x_{2} \leq(1+\nu) x_{1}, x_{2} \leq(1-\nu) x_{1}\right\} \\
\cup\left\{x \in \mathbb{R}^{2} \mid x_{2} \geq(1+\nu) x_{1}, x_{2} \geq(1-\nu) x_{1}\right\} .
\end{array}
$$

Нетрудно убедиться, что для такого конуса $K$ выполнены все условия теоремы 2 при $A=$ $\partial_{2} F(0,0), B=\partial_{2}^{2} F(0,0)$, кроме условия квадратичной аппроксимации, причем $\widetilde{\Sigma}=\mathbb{R}$. Заметим, что здесь не вьполнено ни одно из условий, которьми согласно замечанию 4 можно заменить условие квадратичной аппроксимации в теореме 2. Именно поэтому уравнение

$$
F(\sigma, x)=0
$$

относительно переменной $x$ не имеет решений ни при каком $\sigma \in \mathbb{R} \backslash\{0\}$. 


\section{СПИСОК ЦИТИРОВАННОЙ ЛИТЕРАТУРЫ}

[1] Алексеев В. М., Тихомиров В. М., Фомин С. В. Оптимальное управление. М.: Наука, 1979.

[2] Измаилов А.Ф. О некоторых обобщениях леммы Морса // Тр. МИАН. 1998. Т. 220. C. $142-156$.

[3] Аваков Е.Р. Теоремы об оценках в окрестности особой точки отображения // Матем. заметки. 1990. Т. 47. № 5. С. 3-13.

[4] Иоффе А. Д., Тихомиров В. М. Теория экстремальных задач. М.: Наука, 1974.

[5] Измаилов А.Ф., Третьяков А. А. Факторанализ нелинейных отображений. М.: Наука, 1994.

[6] Аваков Е. Р., Аграчев А. А., Арутюнов А. В. Множество уровня гладкого отображения в окрестности особой точки и нули квадратичного отображения // Матем. сб. 1991. Т. 182. № 8. C. 1091-1104.

[7] Арутюнов А. В. Теорема о неявной функции и анормальные точки // Докл. РАН. 1999. T. 368. № 8. C. 3-5.

Вычислительный Центр РАН, г. Москва

Поступило

E-mail : izmaf@ccas.ru

13.10.1998

Исправленный вариант

07.09.1999 